\title{
Hidradenitis Suppurativa in General Practice: A Pilot Study
}

Farida Benhadou ${ }^{1^{*}}$, Peter Theut Riis ${ }^{2}$, Hassan $\mathrm{Njimi} \mathrm{H}^{3}$, Gregor B.E Jemec ${ }^{2}$, Véronique del Marmol ${ }^{1}$

${ }^{1}$ Department of Dermatology, Erasme Hospital, Université Libre de Bruxelles, Brussels Belgium.

${ }^{2}$ Department of Dermatology, Roskilde Hospital, Denmark.

${ }^{3}$ Department of Biomedical Statistics, Université Libre de Bruxelles, Brussels, Belgium

${ }^{*}$ Corresponding author: Farida Benhadou, Department of Dermatology, Erasme Hospital, Université Libre de Bruxelles, Brussels Belgium, Tel: 0032478810914; E-mail: farida.benhadou@erasme.ulb.ac.be

Rec date: Nov 27, 2015, Acc date: Dec 05, 2015, Pub date: Dec 14, 2015

Copyright: ( 2015 Benhadou F. This is an open-access article distributed under the terms of the Creative Commons Attribution License, which permits unrestricted use, distribution, and reproduction in any medium, provided the original author and source are credited.

\begin{abstract}
Background: Hidradenitis suppurativa (HS) is a chronic debilitating skin disease with a diagnostic delay of 7 years. The general practitioner (GP) is often the first physician consulted by the HS patients, and often provide the initial treatment. Early diagnosis by GP is of major importance and may help to better control the course of the disease.
\end{abstract}

Aim: To describe GP's recognition and management of HS.

Methods: GPs in Belgium and Denmark were invited to complete a questionnaire constructed to describe general knowledge about HS.

Results: 103 Belgian and 51 Danish participated. Demographic characteristics were similar in both groups. Danish GPs estimated the disease to be more common. $0 \% / 21.6 \%$ Belgian/Danish GPs seeing more than 20 patients with $\mathrm{HS}$ per week and conversely $28.2 \% / 3.9 \%$ seeing no patients with HS per week. Belgian GPs were less likely to consider HS a chronic disease than the Danish GPS $(41.7 \% / 84.3 \%)$, and antibiotics were more commonly associated with HS by Danish GPs (31.4\% versus 3.9\%). Interestingly, Belgian and Danish GPs consider HS as an inflammatory (respectively $44.7 \% / 58.8 \%$ ) but also as an infectious disease (respectively $62.1 \% / 64.7 \%$ ).

Conclusions: The early diagnosis and a correct management is a crucial step to improve the prognosis of the disease. This pilot study has attempted to assess the general knowledge about HS of Belgian and Danish GPs. Important differences have appeared, suggesting a need for more information to facilitate the management of the patients. A multidisciplinary approach is suggested for the management of this often debilitating disease. Our pilot study evaluates for the first time the knowledge of GPs about Hidradenitis Suppurativa. Unfortunately the disease is often misdiagnosed and we have reported a mean diagnosis delay of 7 years. An early diagnosis by GPs could greatly improve the course and the management of the disease.

\section{Introduction}

Hidradenitis suppurativa (HS) is a chronic, inflammatory, recurrent, debilitating follicular skin disease that usually presents after puberty with painful, deep-seated, inflamed lesions in the apocrine gland-bearing areas of the body, most commonly the axillary, inguinal and anogenital region (Dessau definition, first International Conference on Hidradenitis suppurativa, March 30-April 1, 2006, Dessau, Germany).

The inflammatory nodules can progress to abscesses and mucopurulent discharge, with hypertrophic scarring and sinus tract formation in the late stages of the disease. HS is often associated with an impaired quality of life $[1,2]$.

HS is histologically characterized by chronic follicular hyperkeratosis and its pathophysiology is not yet fully understood, but it likely includes an interaction between a complex genetic background and the effect of external factors [3].

HS has been considered to be a rare disorder but European studies based on population samples using validated questionnaires have suggested a prevalence of $1 \%-2 \%$ in the general population and a peak prevalence of $4 \%$ (including mild cases) among young adults [4-8].

In the United States, registry studies have suggested a much lower rate of 0.05 to $0 \%, 13 \%[9,10]$. Several factors can play a role in these apparently diverging estimates of the prevalence rate.

Different methodological approaches to the epidemiology undoubtedly play a role, but it may also be speculated that a lack of recognition of HS in the medical community, and reluctance by patients to present their stigmatizing disease for treatment play a role $[11,12]$.

Little is known about the level of recognition of HS in the medical community. The present orphan status of the disease suggests that it is treated by many specialities, often with widely varying understanding of the diseases pathogenesis.

The diagnosis of HS does not require any test and is primarily made on the basis of its typical clinical presentation and has to fit with the criteria adopted by the $2^{\text {nd }}$ International Conference on Hidradenitis suppurativa in San Francisco [13] (Table 1). 
Citation: Benhadou F, Theut Riis P, Njimi HH, Jemec GBE, del Marmol V (2015) Hidradenitis Suppurativa in General Practice: A Pilot Study. J

(1) Typical lesions, i.e., deep-seated painful nodules: 'blind boils' in early lesions; abscesses, draining sinus, bridged scars and 'tombstone' double-ended pseudocomedones in secondary lesions

(2) Typical topography, i.e., axillae, groins, perineal and perianal region, buttocks, infra and inter mammary folds

(3) Chronicity and recurrences

All three criteria must be met for establishing the diagnosis

Table 1: Diagnostic criteria of hidradenitis suppurativa (adopted by the 2nd International Conference on Hidradenitis suppurativa, March 5, 2009, San Francisco, CA US)

The diagnosis has to be discussed when the recurrence of the lesions is more than 2 times/6 months [3].

The general practitioner (GP) is often the first physician consulted by the HS patients, and often provides the initial treatment. Only if initial treatment is not effective will the patients be referred for specialist treatment. Early diagnosis is of major importance and close collaboration between the GP and the specialists can lead to effective control and prevention of advanced disease.

The diagnosis of the disease is often delayed. Globally there appears to be an average delay of 7 years from onset of symptoms to diagnosis. It is speculated that a lack of recognition of the lesions by the physicians may play a role in this [11].
The aim of this study was therefore to evaluate the general awareness of HS among the GPs and comparing the awareness of the Belgian and Danish GPs.

\section{Material and methods}

GPs in Belgium and Denmark were invited to complete a 13 item questionnaire constructed to describe general knowledge about HS (diagnosis, pathophysiology, prevalence, and management). For each question, multiple options were given and the respondent had to choose the most suitable answer(s) according to his/her perception (Table 2).

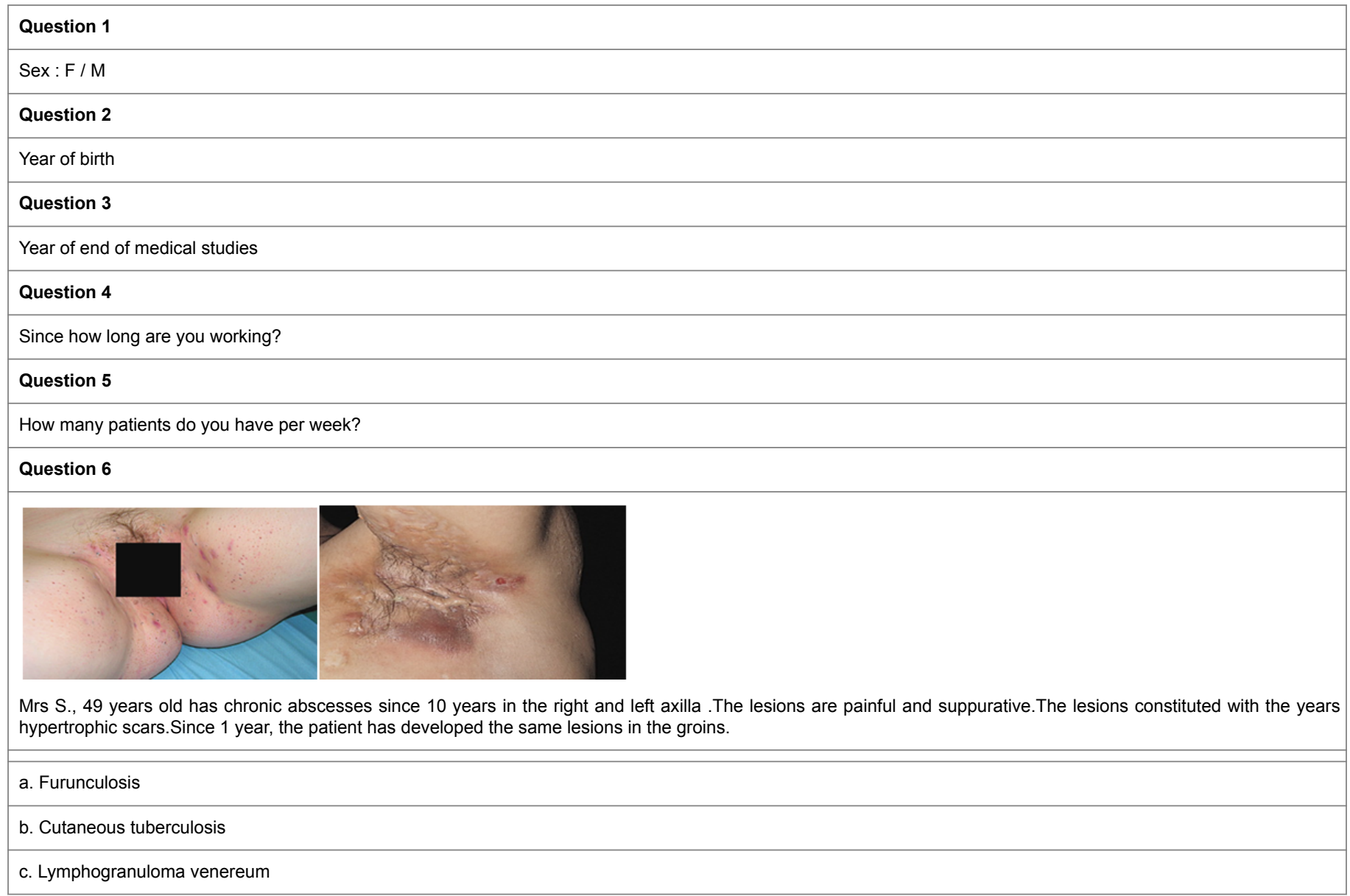


Citation: Benhadou F, Theut Riis P, Njimi HH, Jemec GBE, del Marmol V (2015) Hidradenitis Suppurativa in General Practice: A Pilot Study. J Gen Pract (Los Angel) 3: 207. doi:10.4172/2329-9126.1000207

\section{d. Hidradenitis suppurativa}

e. Abcesses

f. Bacterial infection

\section{Question 7}

Choose the word(s) associated with hidradenitis suppurativa :

a. Chronicity

b. Infection

c. Inflammation

d. Abcesses, furonculosis

e. Handicap

f. Antibiotics

g.Other

Question 8

What is the prevalence of hidradenitis suppurativa in the general population?
a. $1 / 100$
b. $1 / 1000$
c. $1 / 10000$
d. $1 / 500$

\section{Question 9}

How many patients suffering from hidradenitis suppurativa did you see in your career?

a. 0

b.1-5

c. $5-10$

d. $10-20$

e. $>20$

\section{Question 10}

Do you manage hidradenitis suppurativa's patients yourself?

a.Yes

b.No

\section{Question 11}

Do you refer hidradenitis suppurativa's patients to other specialists?

a. Dermatologist

b. Surgeon

c. Internist/ Specialist in infectious disease

d. Other

\section{Question 12}


Citation: Benhadou F, Theut Riis P, Njimi HH, Jemec GBE, del Marmol V (2015) Hidradenitis Suppurativa in General Practice: A Pilot Study. J

\begin{tabular}{|l|}
\hline If you refer to the case in question 6, which treatment do you recommand? \\
\hline a. Topical desinfection and daily bandages \\
\hline b. Topical antibiotics \\
\hline C. Systemic antibiotics \\
\hline d. Surgical excision \\
\hline e. Surgical incision \\
\hline f. Immunosuppressive therapies (TNF blocking agents...) \\
\hline g. Other: \\
\hline Question 13 \\
\hline Do you think that the management of the hidradenitis suppurativa: \\
\hline a. Is easy \\
\hline b. Is complicated \\
\hline c. Needs a multidisciplinary approach \\
\hline
\end{tabular}

Table 2: GPs questionnaire.

A paper format questionnaire was distributed to the Belgian GPs during the annual GPs'meeting organized at the Medical University of Brussels in 2011 (Union des Anciens Etudiants de l'Université libre de Bruxelles) and to the Danish GPs at the Regional Association of GPs meeting in Zealand, Denmark in 2014. In both instances, the questionnaires were collected at the end of the session.

The study was approved by the Ethics Committee of Erasme Hospital,Université Libre de Bruxelles (Reference number : B406.2011.118.56). Questionnaire studies of physicians' knowledge do not require ethical committee approval in Denmark.

\section{Statistical analysis}

Data were analyzed using $\mathrm{IBM}^{\circledR} \mathrm{SPSS}^{\bullet}$ Statistics software, version 22 for Windows. Data are shown as means with standard deviation (SD), medians and interquartile ranges (IQ), or numbers and percentages. Differences between groups of general practitioner (Belgian vs Danish) in distribution of variables were assessed using Student's t-test, MannWhitney test, chi-square test or Fisher's exact test as appropriate. For continuous variables, normality assumption checking was performed by inspection of residual and normal plots. All reported p-values are two-sided and a p-value of less than 0.05 was considered to indicate statistical significance.

\section{Results}

The demographic characteristics of the participants are summarized in (Table 3.1).

\begin{tabular}{|l|l|l|l|}
\hline General practitioner & Belgian & Danish & p-value \\
\hline Number of participants & 103 & 51 & \\
\hline Sex, female, $n(\%)$ & $47(45.6)$ & $20(39.2)$ & 0.45 \\
\hline Age, years, mean \pm sd & $56 \pm 11$ & $55 \pm 7$ & 0.62 \\
\hline
\end{tabular}

\begin{tabular}{|l|l|l|l|}
\hline $\begin{array}{l}\text { Years since medical degree, } \\
\text { median [25\%-75\%] }\end{array}$ & $31[23-37]$ & $23[19-32]$ & 0.005 \\
\hline $\begin{array}{l}\text { Years of practice, median } \\
{[25 \%-75 \%]}\end{array}$ & $28[19-34]$ & $16[11-24]$ & $<0.001$ \\
\hline $\begin{array}{l}\text { Number of patients/week, } \\
\text { median }[25 \%-75 \%]\end{array}$ & $100[70-120]$ & $100[90-120]$ & 0.12 \\
\hline
\end{tabular}

Table 3.1: Demographic characteristics.

In total, 103 Belgian GPs (56 men (54.4\%) and 47 women (45.6\%)) and 51 Danish GPs (31 men (61\%) and 20 women (39\%)) participated in the study. The median age of the participants was similar in both groups. The median of total patients seen by GPs per week did not differ significantly between the groups.

A significant difference between the Belgian and the Danish GPs was observed concerning the number years of practice with respectively 28 , (19years to 34 years ) versus 16 (11-24 years), p-value $<0.001$.

\section{Recognition of HS}

Based on the history and pictures of a clinical case, $86.4 \%$ of the Belgian and $84.3 \%$ of the Danish GPs diagnosed correctly the disease.

Significant differences were observed between the groups with fewer Belgian GPs considering HS a chronic disease (41.7\% versus $84.3 \%$ ) and antibiotics were less frequently associated to HS by the Belgian GPs (3.9\% versus $31.4 \%$ ). The correct prevalence of the disease was well estimated by $6.8 \%$ of the Belgian versus $21.6 \%$ of the Danish GPs.

Differences in the number of HS patients seen per week were also found, with $28.2 \%$ of the Belgian and 3.9\% of the Danish GPs seeing 0 (no patients) and conversely 0 (none) of the Belgian GPs and $21.6 \%$ of the Danish GPs seeing more than 20 patients per week. 
Citation: Benhadou F, Theut Riis P, Njimi HH, Jemec GBE, del Marmol V (2015) Hidradenitis Suppurativa in General Practice: A Pilot Study. J Gen Pract (Los Angel) 3: 207. doi:10.4172/2329-9126.1000207

Page 5 of 7

Belgian and Danish GPs consider HS as an inflammatory (respectively $44.7 \%$ versus $58.8 \%$ ) but also as an infectious disease (respectively $62.1 \%$ versus $64.7 \%$ ).
Finally, $34.4 \%$ of the Belgian and $69.4 \%$ of the Danish GPs managed the HS patients themselves.

Participants' responses are summarized in Table 3.2.

\begin{tabular}{|c|c|c|c|}
\hline General practitioner & Belgian & Danish & p-value \\
\hline 1. Correct diagnosis of hidradenitis suppurativa & $89(86.4)$ & $43(84.3)$ & 0.73 \\
\hline \multicolumn{4}{|l|}{ 2. Characterization of the disease } \\
\hline 2.1 Chronicity & $43(41.7)$ & $43(84.3)$ & $<0.001$ \\
\hline 2.2 Infection & $46(44.7)$ & $30(58.8)$ & 0.1 \\
\hline 2.3 Inflammation & $64(62.1)$ & $33(64.7)$ & 0.76 \\
\hline 2.4 Abcess/furunculosis & $54(52.4)$ & $38(74.5)$ & 0.009 \\
\hline 2.5 Handicap & $29(28.2)$ & $15(29.4)$ & 0.87 \\
\hline 2.6 Antibiotics & $4(3.9)$ & $16(31.4)$ & $<0.001$ \\
\hline 3. Correct prevalence if the disease & $7(6.8)$ & $11(21.6)$ & 0.007 \\
\hline \multicolumn{4}{|l|}{ 4. Number of hidradenitis suppurativa patients/week } \\
\hline 4.10 & $29(28.2)$ & $2(3.9)$ & $<0.001$ \\
\hline $4.21-5$ & $63(61.2)$ & $16(31.4)$ & $<0.001$ \\
\hline $4.35-10$ & $4(3.9)$ & $15(29.4)$ & $<0.001$ \\
\hline $4.410-20$ & $7(6.8)$ & $8(15.7)$ & 0.09 \\
\hline $4.5>20$ & $0(0.0)$ & $11(21.6)$ & $<0.001$ \\
\hline 5.Management of the HS patients by themselves & $35(34.0)$ & $34(69.4)$ & $<0.001$ \\
\hline \multicolumn{4}{|l|}{ 6. Refer to a specialist } \\
\hline 6.1 dermatologist & $86(83.5)$ & $40(78.4)$ & 0.44 \\
\hline 6.2 surgeon & $49(47.6)$ & $19(37.3)$ & 0.22 \\
\hline 6.3 internist & $7(6.8)$ & $0(0.0)$ & 0.1 \\
\hline 6.4 Other & $3(2.9)$ & $0(0.0)$ & 0.55 \\
\hline \multicolumn{4}{|l|}{ 7. Therapeutical options } \\
\hline 7.1 Disinfection & $68(66.0)$ & $7(13.7)$ & $<0.001$ \\
\hline 7.2 Topical antibiotics & $18(17.5)$ & $2(3.9)$ & 0.021 \\
\hline 7.3 Systemic antibiotics & $62(60.2)$ & $33(64.7)$ & 0.59 \\
\hline 7.4 Excision & $38(36.9)$ & $24(47.1)$ & 0.23 \\
\hline 7.5 Incision & $20(19.4)$ & $8(16.0)$ & 0.61 \\
\hline 71.6 Immunosuppressive therapy & $8(7.8)$ & $2(3.9)$ & 0.5 \\
\hline \multicolumn{4}{|l|}{ 8. Management of the disease } \\
\hline 8.1 easy & $6(5.8)$ & $3(5.9)$ & 0.99 \\
\hline 8.2 complicated & $61(59.2)$ & $30(58.8)$ & 0.96 \\
\hline 8.3 recquire multidisciplinary approach & $50(48.5)$ & $20(39.2)$ & 0.27 \\
\hline
\end{tabular}


Table 3.2: How is HS perceived among the belgian and danish general practitioners.

\section{Discussion}

HS is a chronic inflammatory debilitating skin disease and is one of the most distressing conditions observed in dermatology [14-16]. Nevertheless, patients experience a significant delay in diagnosis. We have therefore assessed the level of recognition and self-reported handling of HS by GPs who are often the first physicians to see HS patients in Belgium and Denmark. The data suggests that the disease is well recognized, with an $80 \%$ recognition rate in both countries. The data are self-reported and recognition was based on a constructed case and photographs, and it may be speculated that a different caseconstruction or a more ambiguous case would have changed the recognition rate. The clarity of the case suggests that the reported recognition rate should be regarded as the maximum rate for the respondents.

The diagnosis of HS is primarily clinical, and the key elements required to diagnose HS are the presence of typical and recurrent lesions located in typical skin areas (Table1). HS is a complex disease with various phenotypic expressions and the diagnosis could be easily missed or confused with common infectious skin diseases [1]. Indeed, numerous elements like the common use of antibiotics in HS and the suggested hypothesis of an altered innate and adaptive immunity for its pathogenesis may reinforce the confusion with a skin infection [17]. Interestingly, $\mathrm{HS}$ is often classified in the skin bacterial infection chapter of some handbooks [18]. As potential first line medical care provider, the GP has to be able to recognize the clinical features of the disease and consider the diagnosis in patient with a chronic medical history of painful inflammatory nodules or abcesses with or without discharge. The chronicity of the disease was recognized in both countries, with $84.3 \%$ of the Danish and $41.7 \%$ Belgian GPs describing HS as a chronic disease. In our study, Belgian and Danish GPs consider $\mathrm{HS}$ as an inflammatory (respectively $44.7 \%$ versus $58.8 \%$ ) but also as an infectious disease (respectively $62.1 \%$ versus $64.7 \%$ ) and both propose systemic antibiotics as a primary therapeutic option to manage the disease, but although they also consider HS to be an inflammatory disorder only $7,8 \%$ of the Belgian and $3,9 \%$ of the Danish consider immunosuppressive therapy. A flare of the disease is frequently characterized by painful inflammatory nodules, abscesses and/or discharge. Based on our experience, antibiotics and/or incision are often proposed by general physicians with the hypothesis of a bacterial infection. We observed in our pilot study that the Belgian GPs propose more frequently incisions than Danish GPs (20\% versus $8 \%$ ).

HS is a complex and heterogenous disease and its treatment remains a major challenge in general practice. In our work, Belgian GPs less frequently manage the HS patients themselves (34, $4 \%$ versus $69,4 \%$ respectively). Frequently considered as gate-keepers to specialized health-care providers, a dynamic interplay between GPs and specialist is therefore suggested for the management of HS. GPs could play a key role in the early diagnosis and follow-up of the HS patients and will undoubtedly always be the link between the patient and the specialist.

The strength of our work is that is the first pilot study evaluating the general knowledge of GPs. The study was conducted among the Belgian and Danish GPs where the highest prevalence of HS has been reported in Denmark. We are planning to continue and include many other countries.

The weakness of our exploratory study is the paper format of the questionnaire, the sample size and the sampling procedure. The paper format did not allow the data to be divulged successively, i.e. Participants could turn to the last pages of the questionnaire where they could find the words "Hidradenitis suppurativa". Participants were instructed to fill in the questionnaire consecutively, but not all may have done so and it is unknown to what extents this may have influence the answers. An electronic version may therefore be preferable in future studies. The sampling was done based on available samples at meetings and therefore suffers from potential bias and limitations of size. The meetings were, however, not related to the subject matter (skin disease).

\section{Conclusions}

The early diagnosis and a correct management is a crucial step to improve the prognosis of the disease. GPs are often the first physicians visited by the HS patients. This pilot study has attempted to assess for the first time the general knowledge about HS of Belgian and Danish GPs. Important differences have appeared that may derive for the number of patients seen by each. GP's awareness is still limited and may play a role in the significant delay experienced by HS patients. A multidisciplinary approach is highly recommended for the management of this often debilitating disease.

\section{References}

1. Jemec GB (2012) Clinical practice: Hidradenitis Suppurativa. New England Journal of Medicine 366: 158-164.

2. Esmann S, Jemec GB (2011) Psychosocial impact of hidradenitis suppurativa: a qualitative study. Acta Derm Venereol 91: 328-332.

3. Zouboulis CC, N. Desai N, Emtestam L, Hunger RE, D. Ioannides, et al. (2015) European S1 guideline for the treatment of hidradenitis suppurativa/acne inversa. J Eur Acad Dermatol Venereol 29: 619-644.

4. Jemec GB, Heidenhem M, Nielsen NH (1996) The prevalence of HS and its potential precursor lesions.J Am Acad Dermatol 35:191-194.

5. Revuz JE, , Canoui-Poitrine F, Wolkenstein P, Viallette C, Gabison G, et al. (2008) Prevalence and factors associated with hidradenitis suppurativa: results from two case-control studies. J Am Acad Dermatol 59: 596-601.

6. Vinding GR, Miller IM, Zarchi K, Ibler KS, Ellervik C, et al. (2014) The prevalence of inverse recurrent suppuration: a population-based study of possible hidradenitis suppurativa. Br J Dermatol 170: 884-889.

7. Pascoe VL, Kimball AB (2014) Hidradenitis suppurativa: current progress and future questions. JAMA Dermatol.150: 1263-1264

8. Jemec GB, Heidenhem M, Nielsen NH (1998) Prevalence of HS in Denmark.Ugeskr Laeger 160:847-849

9. Shahi V, Alikhan A, Vazquez BG, Weaver AL, Davis MD, et al. (2014) Prevalence of Hidradenitis Suppurativa: A Population-Based Study in Olmsted County, Minnesota.Dermatol 229: 154-158 .

10. Cosmatos I, Matcho A, Weinstein R, Montgomery MO, Stang P, et al. (2013) Analysis of patient claims data to determine the prevalence of hidradenitis suppurativa in the United States.J Am Acad Dermatol 68: 421-429.

11. Saunte M, Boer J, Stratigos A, Szepietowski JC, Hamzavi I et al. (2015) Diagnostic delay in Hidradentiis suppurativa. Br J Dermatol. 
Citation: Benhadou F, Theut Riis P, Njimi HH, Jemec GBE, del Marmol V (2015) Hidradenitis Suppurativa in General Practice: A Pilot Study. J Gen Pract (Los Angel) 3: 207. doi:10.4172/2329-9126.1000207

Page 7 of 7

12. Jemec GB, Kimball A (2015) Hidradenitis suppurativa: Epidemiology and scope of the problem. J Am Acad Dermatol 73: S4-S7

13. Hidradenitis suppurativa foundation.

14. Dufour D, Emtestam L, Jemec GB (2014) Hidradenitis suppurativa: a common and burdensome, yet under-recognised, inflammatory skin disease. Postgrad Med J 90: 216-221.

15. Wolkenstein P, Loundou A, Barrau K, Auquier P, Revuz J (2007) Quality of Life Group of the French Society of Dermatology. Quality of life impairment in hidradenitis suppurativa: a study of 61 cases. J Am Acad Dermatol 56: 621-623.
16. Shlyankevich J, Chen AJ, Kim GE, Kimball AB (2014) Hidradenitis suppurativa is a systemic disease with substantial comorbidity burden: a chart-verified case-control analysis.J Am Acad Dermatol 71: 1144-1150.

17. Ring HC, Riis Mikkelsen P, Miller IM, Jenssen H, Fuursted K, et al. (2015) The bacteriology of hidradenitis suppurativa: a systematic review. Exp Dermatol. 10: 727-731.

18. Creamer D, Barker J, Kerdal F. Acute Adult Dermatology, Handbook 2. 